\title{
Avaliação in vitro do fator de proteção solar (FPS) de cosméticos fotoprotetores manipulados
}

\author{
In vitro evaluation of sun protection factor (SPF) \\ of manipulated sunscreen cosmetics
}

Recebido em: $13 / 08 / 2018$ Aceito em: $\quad 15 / 12 / 2018$
Gabriela Gislaine SECCO; Betina Montanari BELTRAME; Melissa SCHWANZ Universidade de Caxias do Sul, Área do Conhecimento de Ciências da Vida. Rua Ernesto Zaparolli, 496, CEP 95059-848. Caxias do Sul, RS, Brasil. E-mail: bmbeltrame@ucs.br

\section{ABSTRACT}

Aiming to reduce the harmful effects caused on the skin by solar radiation, the use of photoprotectors is a reality these days. Based on the importance of the subject, the objective of this study was to experimentally determine by spectrophotometry in the ultraviolet region the solar protection factor (SPF); to evaluate the macroscopic and physicochemical characteristics of manipulated photoprotectors labeled SPF 30; evidencing the possibility of SPF determination by the in vitro technique as a quality control for compounding pharmacies. The results showed that the formulations appeared to be homogeneous, with normal organoleptic characteristics. The $\mathrm{pH}$ remained in the range of 6.30 to 7.66. In the centrifugation test, one of the samples presented slight evidence of inhomogeneity and the others did not show any change in relation to their original appearance. Regarding the SPF in vitro, one of the formulations under evaluation presented a value above the labeling and the others presented lower SPF than those declared on the labels. It is evidenced that the in vitro technique used in the work can contribute to the determination and control of manipulated products since it is a reliable technique.

Key-words: sun protection factor; photoprotectors; in vitro evaluation

\section{RESUMO}

Visando reduzir os efeitos nocivos causados na pele pela radiação solar, a utilização de fotoprotetores é uma realidade. Com base na importância do tema, o objetivo dessa pesquisa foi determinar experimentalmente, por espectrofotometria na região do ultravioleta, o fator de proteção solar (FPS) e, ainda, avaliar características macroscópicas e físico-químicas de fotoprotetores manipulados rotulados FPS 30, além de evidenciar a possibilidade de determinação do FPS pela técnica in vitro para controle da qualidade em as farmácias magistrais. Os resultados mostraram que as formulações se apresentaram visivelmente homogêneas, de coloração normal, odor característico e toque agradável. O pH manteve-se na faixa de 6,30 a 7,66. No teste de estabilidade por centrifugação uma das amostras apresentou leve evidência de falta de homogeneidade e as demais não apresentaram qualquer alteração em relação ao seu aspecto original. Quanto ao FPS in vitro, uma das formulações em estudo obteve valor acima do rotulado e as demais obtiveram FPS inferiores aos declarados nos rótulos. A avaliação in vitro utilizada no trabalho pode contribuir para a determinação e controle de produtos manipulados, por ser uma técnica confiável e de fácil execução.

Palavras-chave: fator de proteção solar; fotoprotetores; avaliação in vitro 


\section{INTRODUÇÃO}

A radiação solar é um elemento essencial para a vida. Dentre as ações benéficas da radiação solar, podem ser destacadas a influência positiva sobre o humor, levando a uma sensação de bem estar, síntese de Vitamina D e estímulo à produção de melanina com consequente bronzeamento da pele, atualmente um padrão de beleza. Porém, as mesmas radiações solares que trazem benefícios podem causar danos à pele humana, dependendo da duração e frequência de exposição, intensidade das radiações e da sensibilidade do indivíduo (1-3).

A radiação solar é subdividida em três faixas: radiação ultravioleta $\mathrm{C}$ (UVC), radiação ultravioleta B (UVB) e radiação ultravioleta A (UVA). Os efeitos da radiação solar estão diretamente relacionados com o tipo de radiação, sendo que a energia aumenta com a redução do comprimento de onda. A radiação UVC (100-290 nm) é altamente lesiva ao homem, no entanto é absorvida pela camada de ozônio antes de atingir a superfície terrestre. (4-7)

A radiação UVB (290-320 nm), por sua vez, representa 5\% das radiações ultravioleta (UV) que atingem a Terra. Possui alta energia, sendo responsável pelos danos agudos e crônicos a pele, tais como manchas, queimaduras, descamação e envelhecimento precoce das células. A exposição frequente e intensa à radiação UVB pode causar lesões no DNA, além de suprimir a resposta imunológica da pele, aumentando o risco de mutações fatais e podendo levar ao desenvolvimento de câncer de pele. A radiação UVA (320-400 nm) é a mais abundante, representando cerca de 95\% das radiações UV que atingem a Terra, porém é a radiação menos energética. Atinge a derme profunda, afetando negativamente a elasticidade natural da pele, sendo responsável pelo envelhecimento cutâneo precoce. (4-7)

A busca por proteção contra a radiação solar iniciou-se efetivamente, nas duas últimas décadas quando os efeitos nocivos do sol se tornaram mais conhecidos e divulgados. O rápido crescimento da comercialização de produtos contendo filtros solares indica que as pessoas estão conscientes dos possíveis perigos do fotoenvelhecimento e risco de desenvolvimento de câncer de pele, que ocorrem como resultado da superexposição ao sol, principalmente em horários inadequados $(8,9)$.

A necessidade do uso de protetores solares, também conhecidos como fotoprotetores, é uma realidade indiscutível nos dias atuais. Os filtros solares são substâncias químicas com propriedades de absorver, refletir e dispersar a radiação que incide sobre a pele. A eficácia de um filtro solar é medida em função de seu fator de proteção solar, FPS, o qual indica quantas vezes o tempo de exposição ao sol, sem o risco de eritema, pode ser aumentado com uso de protetor solar. Para avaliar o FPS são utilizadas técnicas in vivo e in vitro. A metodologia in vitro, se baseia nas propriedades absortivas ou refletoras do filtro, sendo um método bastante eficaz para determinação do FPS, enquanto que a metodologia in vivo utiliza voluntários humanos, sendo a técnica utilizada para registro de tais cosméticos. $(1,10-12)$

A ciência dos efeitos prejudiciais da radiação solar observada nas últimas décadas tem estimulado o interesse em novas tecnologias de proteção solar, levando ao desenvolvimento de filtros inovadores, visto que os consumidores têm buscado níveis de proteção mais altos, sendo a qualidade dos fotoprotetores um fator crucial para a prevenção dos efeitos danosos da radiação. A determinação do FPS indica a eficácia dos filtros solares para a porção UVB do espectro eletromagnético, região responsável por causar eritema na pele. Um fotoprotetor eficaz é aquele capaz de proteger a pele exposta à radiação solar, sendo que quanto maior for o FPS maior será a proteção frente ao eritema solar. (13-15)

As farmácias, diferentemente das indústrias cosméticas, não precisam comprovar a eficácia dos fotoprotetores; podem manipular e estimar os valores de FPS, por meio de softwares que calculam a quantidade de filtros a serem utilizados para determinado FPS. A realização do teste in vivo para comprovação do FPS é inviável para as farmácias de manipulação, devido ao elevado custo e às diferentes formulações manipuladas. Assim sendo, os consumidores questionam a eficácia e seguranças das formulações manipuladas contendo filtros solares. $(16,17)$

Com base na importância do tema, o objetivo dessa pesquisa foi determinar experimentalmente 
por espectrofotometria na região do ultravioleta $\mathrm{o}$ fator de proteção solar e, ainda, avaliar características macroscópicas e físico-químicas de fotoprotetores manipulados com FPS rotulado igual a 30 obtidos em estabelecimentos farmacêuticos da cidade de Caxias do Sul (RS). Pretendeu-se também evidenciar a possibilidade de determinação do FPS pela técnica in vitro como um controle de qualidade para as farmácias magistrais.

\section{MATERIAL E MÉTODOS}

Formulações. Foram obtidas 10 amostras de 10 farmácias da cidade de Caxias do Sul (RS), rotuladas com FPS 30, na forma de gel-creme, não contendo filtros físicos na composição, visto que estes são insolúveis no solvente utilizado na técnica in vitro utilizada neste estudo.

Análise macroscópica. A análise macroscópica foi realizada por meio da determinação de características sensoriais e uniformidade das formulações a fim de identificar possíveis processos de instabilidade. Avaliou-se o aspecto, cor, odor e sensação ao toque das formulações (18).

Teste de centrifugação. A avaliação frente à centrifugação foi realizada empregando $5 \mathrm{~g}$ da amostra e centrifugando a $3000 \mathrm{rpm}$ por $30 \mathrm{~min} \mathrm{em}$ centrífuga (Cientec, modelo CT 6000) (19).

Determinação do valor de $\mathbf{p H}$. Para determinação do $\mathrm{pH}$ as amostras foram diluídas em água destilada (pH 7,0) até a obtenção de solução com concentração de $10 \%(\mathrm{~m} / \mathrm{v})$. As determinações foram realizadas em triplicata em peagômetro digital (Micronal ${ }^{\circledR}$ B474) previamente calibrado (20).

Determinação do Fator de Proteção Solar (FPS). Para a determinação do FPS utilizou-se o método espectrofotométrico in vitro desenvolvido por Mansur e cols. (1986) (21).

Preparo das amostras. Para o preparo das amostras foi pesado cerca de $0,5 \mathrm{~g}$ dos fotoprotetores, transferido para balão volumétrico de 100 $\mathrm{mL}$, contendo cerca de $30 \mathrm{~mL}$ de etanol $96{ }^{\circ} \mathrm{GL}$. A amostra foi solubilizada e o volume completado com o mesmo solvente. Uma alíquota de 1,0 mL foi transferida para balão volumétrico de $25 \mathrm{~mL}$, completando o volume com etanol $96^{\circ} \mathrm{GL}$ e homo- geneizando o conteúdo, obtendo uma solução com concentração final de $0,2 \mathrm{mg} / \mathrm{mL}(8,11)$.

Leitura das amostras. A análise foi realizada em triplicata, a partir de uma solução amostra preparada com cada formulação, e as absorvâncias das soluções amostras foram medidas em espectrofotômetro (Thermo Scientific GENESYS) no intervalo de 290 - 320, faixa correspondente à radiação UVB responsável por causar eritema na pele, a cada 5 nm, logo após a diluição de cada produto. (21).

Cálculo do Fator de Proteção Solar (FPS). Para o cálculo do FPS foi utilizada a expressão matemática descrita por Mansur e cols (1986) (21). A relação entre o efeito erimatogênico e a intensidade da luz de cada comprimento de onda foi determinada conforme escrito por Sayre e cols (1979) (22) e mostrado na Tabela 1.

$$
F P S=F C \cdot \sum_{290}^{320} E E(\lambda) . I(\lambda) \cdot a b s(\lambda)
$$

Em que:

$\mathrm{FC}=$ Fator de correção (10);

$\mathrm{EE}(\Lambda)=$ efeito erimatogênico da radiação em cada comprimento de onda $\Lambda$;

$\mathrm{I}(\Lambda)=$ intensidade da radiação solar em cada $\Lambda$; Abs $=$ Absorvância em cada $\Lambda$.

Análise dos dados. Os resultados foram dados como média aritmética \pm o valor do desvio padrão (média \pm DP) e comparados através de análise de variância (ANOVA), seguido do teste de Dunnet, considerando valores de $\mathrm{p}<0.01$ como significativos.

Tabela 1. Relação efeito eritemogênico conforme o comprimento de onda $(\gamma)(22)$.

\begin{tabular}{|c|c|c|}
\hline$(y) n m$ & EE.I & I \\
\hline 290 & 0,0150 & \\
\hline 295 & 0,0817 & \\
\hline 300 & 0,2874 & \\
\hline 305 & 0,3278 & \\
\hline 310 & 0,1864 & \\
\hline 315 & 0,0839 & \\
\hline 320 & 0,0180 & \\
\hline$\Sigma$ & 1,0000 & \\
\hline
\end{tabular}




\section{RESULTADOS E DISCUSSÃO}

Por uma questão ética, a identidade das farmácias foi preservada. Para facilitar a apresentação dos resultados, estes foram identificados por números.

Análise Macroscópica. Os resultados da análise macroscópica encontram-se descritos no Quadro 1.

Teste de Centrifugação. A formulação 3 apresentou um sinal de instabilidade após ser submetida à centrifugação por 30 minutos à $3000 \mathrm{rpm}$. As demais formulações não apresentaram qualquer alteração em relação ao seu aspecto original, permanecendo estáveis nesse parâmetro.

Determinação de pH. Os resultados das avaliações do $\mathrm{pH}$ das formulações estudadas estão representados na Tabela 2.

Quadro 1. Caracterização macroscópica de fotoprotetores manipulados.

\begin{tabular}{|c|c|c|c|c|}
\hline Amostras & Aspecto & Cor & Odor & Toque \\
\hline 1 & NS & N & N & A \\
\hline 2 & NS & N & N & A \\
\hline 3 & NS & N & N & A \\
\hline 4 & NS & N & N & A \\
\hline 5 & NS & N & N & A \\
\hline 6 & NS & N & N & A \\
\hline 7 & NS & N & N & D \\
\hline 8 & NS & N & N & D \\
\hline 9 & NS & N & N & A \\
\hline 10 & NS & N & N & A \\
\hline
\end{tabular}

Aspecto: NS - Não há separação da fase oleosa; S - separação da fase oleosa. Cor: N - Normal; M - Modificado; IM - intensamente modificado. Odor: N - Normal; M - Modificado; IM - intensamente modificado. Toque: A - toque agradável, fácil aplicação na pele; D - toque desagradável, pegajoso; $M D$ - toque muito desagradável, muito pegajoso, compromete a aplicação na pele.
Tabela 2. Valores de pH e de fator de proteção solar (FPS) de fotoprotetores manipulados.

\begin{tabular}{ccc} 
amostra & pH & FPS \\
\hline 1 & $6,94 \pm 0,010$ & $20,893 \pm 0,123$ \\
2 & $7,07 \pm 0,015$ & $35,247 \pm 0,107$ \\
3 & $6,76 \pm 0,015$ & $17,437 \pm 0,049$ \\
\hline 4 & $6,3 \pm 0,010$ & $27,003 \pm 0,054$ \\
\hline 5 & $7,08 \pm 0,104$ & $21,956 \pm 0,013$ \\
6 & $6,77 \pm 0,017$ & $21,733 \pm 0,080$ \\
\hline 7 & $7,66 \pm 0,044$ & $18,756 \pm 0,036$ \\
8 & $6,70 \pm 0,015$ & $8,878 \pm 0,058$ \\
9 & $7,38 \pm 0,006$ & $19,145 \pm 0,067$ \\
10 & $7,06 \pm 0,0111$ & $23,308 \pm 0,077$ \\
\hline
\end{tabular}

Determinação do Fator de Proteção Solar (FPS). Segundo a metodologia proposta por Mansur e cols (1986), as absorvâncias obtidas nas leituras espectrofotométricas no intervalo de 290 a $320 \mathrm{~nm}$ foram aplicadas na fórmula dos referidos autores (21). Os resultados do cálculo do FPS estão mostrados na Tabela 2.

Os resultados foram expressos como média \pm DP em triplicata. A comparação estatística foi realizada por ANOVA seguida pelo teste de Dunnett. ${ }^{*} \mathrm{p}<0.01$ comparado ao valor referência (FPS 30).

As características organolépticas permitiram avaliar qualitativamente o estado em que se encontravam as amostras em estudo, com o objetivo de verificar alterações no que se refere à cor, odor e aparência, parâmetros que influenciam a aceitação pelo consumidor $(19,23,24)$. Todas as formulações em estudo apresentaram-se aparentemente homogêneas, com coloração do branco ao amarelo claro e odor característico. Com relação ao aspecto sensorial, as formulações 7 e 8 apresentaram um toque desagradável e as demais, toque agradável e fácil aplicação. 
No teste de centrifugação, a força da gravidade atua sobre a amostra fazendo com que suas partículas se movam no seu interior, assim produzindo estresse e simulando um aumento na força da gravidade, elevando a mobilidade das partículas e antecipando possíveis instabilidades. Assim sendo, este teste permite verificar a estabilidade da formulação, possibilitando observar rapidamente a separação de fases da dispersão, avaliando a coalescência ou a cremeação. Isso permite prever se o produto perderá a homogeneidade de fases em função do tempo. A instabilidade dos fotoprotetores está relacionada à presença de maior número de filtros na composição $(25,26)$. A formulação 3 apresentou sinal de instabilidade, manifestada pelo fenômeno de cremeação, sendo uma constatação importante, pois denota que a formulação deve ser analisada criteriosamente em relação a sua composição, a fim de obter um produto mais estável e de qualidade. As demais formulações expostas à centrifugação permaneceram estáveis, não apresentando qualquer alteração em relação ao seu aspecto original.

Em relação ao $\mathrm{pH}$, para ser obtida uma formulação de qualidade, esta deve ser quimicamente neutra ou ligeiramente ácida, não devendo ultrapassar $\mathrm{pH} 7,5$, sendo importante para a estabilidade de cosméticos fotoprotetores, uma vez que os filtros solares químicos são mais estáveis em $\mathrm{pH}$ próximos a neutralidade. Além disso, resulta em um produto compatível com a aplicação cutânea, cuja epiderme possui valores de pH fisiológico (4 a 6,5). Alterações relacionadas ao valor de $\mathrm{pH}$ químico são parâmetros que podem estar associados a reações de hidrólise ou oxidação dos filtros solares $(26,27$, 28). Os valores de $\mathrm{pH}$ das formulações avaliadas nesse estudo mantiveram-se na faixa de 6,30 a 7,66, indicando grande semelhança nos valores entre as formulações. As formulações 7 e 10 ficaram um pouco acima do recomendado para fotoprotetores, podendo alterar o $\mathrm{pH}$ natural da pele causando um desequilíbrio, e consequentemente alergias ou irritações. A pele é formada por queratina e pode ter a estrutura desnaturada em $\mathrm{pH}$ alto, ocasionando a quebra da estrutura dimensional da proteína.

A eficácia de um fotoprotetor é caracterizada pelo seu FPS, o qual, teoricamente, é definido como a razão entre a quantidade de radiação solar necessária para produzir uma dose eritematogênica mínima na pele protegida pelo filtro solar e na pele não protegida. Na prática, significa o tempo que o indivíduo poderá se expor ao sol, usando protetor, sem apresentar o eritema solar $(26,29)$.

O FPS das formulações em estudo foi determinado por método espectrofotométrico (21) largamente empregado por outros autores $(8,11,23$, 30-36). Esse método é realizado por meio de espectrofotometria na região UV e avalia o filtro solar de acordo com a altura, largura e localização da sua curva de absorção dentro do espectro do ultravioleta. Essa técnica tem mostrado ser rápida e eficaz, além de apresentar uma boa correlação com os resultados in vivo, o que garante a confiabilidade da técnica. Esse método pode auxiliar na triagem durante o desenvolvimento de cosméticos fotoprotetores, facilitando o controle da qualidade das mesmas $(8,21,37-42)$.

Em relação ao FPS in vitro observado nas análises realizadas, apenas a formulação 2 apresentou um FPS acima do rotulado na embalagem. Todas as demais formulações apresentaram valores de FPS inferiores aos declarados nos rótulos. As formulações 4 e 10, embora não tenham atingido o FPS informado, apresentaram mais de $90 \%$ do fator especificado no rótulo, porém não existem especificações na literatura de limites de variação para a metodologia in vitro.

Foi observado, ainda, pela análise estatística, que se excetuando a formulação 10 , todas as outras tiveram valores significativamente diferentes do valor referência ou evidenciado nos rótulos dos produtos (FPS 30). Alguns estudos também reportam valores diferentes de FPS dos descritos nos rótulos $(8,14,17,23,32,43,44)$, demonstrando a importância de se verificar o FPS in vitro como um controle de qualidade para as farmácias, a fim de garantir a eficácia e segurança do produto.

Diversos fatores podem interferir no FPS de uma formulação, como a escolha dos filtros utilizados, método de preparação, matérias-primas empregadas, em especial os emolientes, emulsionantes, solventes e ativos utilizados, fotoestabilidade e cristalização dos filtros solares $(8,23,45-49)$.

A concentração e o tipo de filtro exercem um efeito direto sobre o grau de proteção obtido. A efi- 
cácia dos filtros é diferente entre si, podendo alguns ser mais efetivos que outros, sendo necessária a combinação de dois ou mais filtros para alcançar um FPS desejado. Os filtros devem ficar retidos na superfície da pele para serem eficazes, bem como formar um filme sobre a pele e permear o mínimo possível para a circulação sistêmica, visto que a fotoproteção é perdida se o filtro é absorvido $(45,50)$.

Os fotoprotetores em estudo possuiam em sua composição apenas filtros químicos, precisando dessa forma utilizar uma maior concentração destes para atingir um FPS elevado. Segundo alguns estudos, a tendência é a redução na concentração de filtros químicos ou utilização de novas tecnologias, como o microencapsulamento para incorporação dos filtros orgânicos nas formulações, devido aos eventos adversos ocasionados pelo uso tópico destas substâncias e ao mesmo tempo pela sua absorção cutânea, o que compromete a eficácia do fotoprotetor $(1,6,7,12,49,51)$.

A utilização da técnica de espectrofotometria pode ser um importante parâmetro para avaliar a eficácia de fotoprotetores manipulados, podendo ser

\section{REFERÊNCIAS}

1. Flor J, Davolos MR, Correa MA. Protetores Solares. Quim Nova 2006; 30(1): 153-158. DOI: 10.1590/S010040422007000100027

2. Silva KMO, Ferrari M. Determinação in vitro do fator de proteção solar de formulações de farmácias magistrais. Infarma 2007; 19(5/8): 125-130.

3. Ribeiro CJ. Cosmetologia aplicada a dermoestética. 2 ed. São Paulo: Pharmabooks; 2010.

4. Gomes RK, Damazio MG. Cosmetologia: descomplicando os princípios ativos. 3 ed. São Paulo: LMP; 2009

5. Schalka S. Influência na quantidade aplicada de protetores solares no Fator de Proteção Solar (FPS): avaliação de dois protetores solares com os mesmos ingredientes em diferentes concentrações [Dissertação]. São Paulo: Faculdade de Medicina, Universidade de São Paulo, 2009.

6. Balogh TS, Velasco MV, Pedriali CA, Baby AR. Ultraviolet radiation protection: current available resources in photoprotection. An Bras Dermatol 2011; 86(4):732-42. DOI: $10.1590 / \mathrm{S} 0365-05962011000400016$

7. Teixeira SMMCG. Veiculação de filtros solares utilizados na fotoproteção. [Dissertação]. Porto: Faculdade de Ciências da Saúde, Universidade Fernando Pessoa, 2012. utilizada pelas farmácias como um controle da qualidade de suas formulações. Para isso, é necessário que a técnica seja validada e realizada com vidrarias e equipamentos calibrados a fim de obterem valores mais exatos com desvios padrões mais baixos.

\section{CONCLUSÃO}

A partir dos resultados obtidos é possível perceber que se faz necessária a avaliação do real fator de proteção das formulações que são preparadas em farmácias de manipulação, a fim de garantir a eficácia e a qualidade dos produtos que estão sendo comercializados. Ainda, a técnica in vitro utilizada no trabalho pode contribuir para a determinação e controle de produtos manipulados, por ser uma técnica confiável e de fácil execução.

\section{AGRADECIMENTOS}

Os autores agradecem às farmácias que contribuíram para o estudo e à Universidade de Caxias do Sul..
8. Dutra EA, Oliveira DAGC, Hackmann ERMK, Santoro MIRM. Determination of sun protection factor (SPF) of sunscreens by ultraviolet spectrophotometry. Rev Bras Cienc Farm 2004; 40(3): 381-385. DOI: 10.1590/S151693322004000300014

9. Silva BS, Carvalho CPA, Vicentini ÉS, Cole ER. Efeitos da bioacumulação de filtros solares com atividade estrogênica na saúde da população. Infarma 2009; 21(7): 3845.

10. BRASIL. Agência Nacional de Vigilância Sanitária. Resolução RDC nº. 237, de 22 de agosto de 2002. Aprova o Regulamento Técnico Sobre Protetores Solares em Cosméticos. Diário Oficial da União, $\mathrm{n}^{\circ} 163$, de 23 de agosto de 2002. Seção 1E. p. 40.

11. Ribeiro RP, Santos VM, Medeiros EC, Silva VA, Volpato NM, Garcia S. Avaliação do Fator de Proteção Solar (FPS) in vitro de produtos comerciais e em fase de desenvolvimento. Infarma 2004; 16(4): 85-88.

12. Velasco MRV, Balogh TS, Pedriali CA, Sarruf FD, Pinto CASO, Kaneko TM, Baby AR. Associação da rutina com p-metoxicinamato de octila e benzofenona-3: avaliação in vitro da eficácia fotoprotetora por espectrofotometria de refletância. Lat Am J Pharm 2008;27(1):23-27. 
13. Hojerováa J, Medovcíkováa A, Mikulab M. Photoprotective efficacy and photostability of fifteen sunscreen products having the same label SPF subjected to natural sunlight. Int J Pharm 2011; 408(1-2): 27-38. DOI: 10.1016/j. ijpharm.2011.01.040

14. Roca LM, Moreira S, Moreira LMA. Avaliação laboratorial do fator de proteção solar (FPS) em protetores utilizados por portadores de albinismo na Bahia. Rev Cienc Med Bio 2011; 10(2): 136-139. DOI: 10.9771/cmbio. v10i2.5447

15. Criado PR, Melo JN, Oliveira ZNP. Topical photoprotection in childhood and adolescence. J Pediatr 2012; 88(3): 203-210. DOI: 10.2223/JPED.2194

16. Silva CF. Testes para avaliação do fator de proteção solar de produtos cosméticos fotoprotetores [Monografia]. São Paulo: Curso de Farmácia, Centro Universitário das Faculdades Metropolitanas Unidas; 2007.

17. Ferreira GM, Moraes AJ. Avaliação in vitro da eficácia de fotoprotetores preparados em farmácias magistrais na cidade de Uberlândia - MG. E-Rac 2013; 3(1).

18. Vieira RP, Fernandes AR, Kaneko TM, Consiglieri VO, Pinto CASO, Pereira CSC, Baby AR, Velasco MVR. Physical and physicochemical stability evaluation of cosmetic formulations containing soybean extract fermented by Bifidobacterium animalis. Braz J Pharm Sci 2009; 45(3): 515-525.

19. BRASIL. Agência Nacional de Vigilância Sanitária. Guia de estabilidade de produtos cosméticos. v.1. Brasília; 2004.

20. BRASIL. Agência Nacional de Vigilância Sanitária. Farmacopeia Brasileira. v. 1. 5. ed. Brasília, 2010. 523 p.

21. Mansur JS, Breder MNR, Mansur MCA, Azulay RD. Determinação do fator de proteção solar por espectrofotometria. An Bras Dermatol 1986; 61(3):121-124.

22. Sayre RM, Agin PP, Levee GJ, Marlowe E. A comparison of in vivo and in vitro testing utilization of sunscreening formulas. Photochem Photobiol 1979; 29(3): 559-566. DOI: 10.1111/j.1751-1097.1979.tb07090.x.

23. Alves LA, Pereira JA, Ferreira RC, Gomes, AJPS. Avaliação in vitro do fator de proteção solar a partir de fotoprotetores manipulados contendo agentes ativos antienvelhecimento. Colloquium Vitae 2010; 2(2): 50-56. DOI: 10.5747/cv2010.v02.n2.v038

24. Moraes GG, Santos ODH, Masson DS. Development of $\mathrm{O} / \mathrm{W}$ emulsions with annato oil (Bixa orellana) containing liquid crystal. J Dispers. Sci Techn, 26: 591-6, 2005. DOI: $10.1081 /$ DIS-200057647

25. Silva EC, Soares IC. Tecnologia de emulsões. Cosmet Toiletries 1996; 8(5): 37-46.

26. Singh MV, Gomes JP, Guimarães PR, Baranhuk BF. Estabilidade de Emulsões Magistrais com FPS. Cosmet Toiletries 2006; 18: 66-70.
27. Block L.H. Medicação tópica. In: Gennaro, AR. Remington: the science and practice of pharmacy. 20. ed. Philadelphia: Lippincott Willians \& Wilkins, 2004.

28. Souza VM. Ativos dermatológicos. 2.ed. São Paulo: Tecnopress, 2004.

29. Nagashima N, Hanada K, Hashimoto I. Correlation of skin phototype with facial wrinkle information. Photodermatol Photoimmunol Photomed 1999; 15(1): 2-6. DOI: 10.1111/j.1600-0781.1999.tb00044.x.

30. Barth AL, Santos EP, Garcia S, Rezende VP. Análise e seleção de filtros solares químicos com alta qualidade em proteção solar. Riopharma 1999: 25-27.

31. Santos EP, Freitas ZM, Souza KR, Garcia S, Vergnanini A. In vitro and in vivo determinations of sun protection factors of sunscreen lotions with octylmethoxycinnamate. Int J Cosmet Sci 1999; 21(1): 1-5. DOI: 10.1046/j. 1467-2494.1999.181658.x.

32. Borghetti GS, Knorst MT. Desenvolvimento e avaliação da estabilidade física de loções O/A contendo filtros solares. Rev Bras Cienc Farm 2006;42(4):531-537.

33. Ferrari M, Oliveira MSC, Nakano AK, Rocha-Filho PA. Determinação do fator de proteção solar (FPS) in vitro e in vivo de emulsões com óleo de andiroba (Carapa guianensis). Rev Bras Farmacogn 2007; 17(4):626-630. DOI: 10.1590/S0102-695X2007000400023

34. Violante IMP, Souza IM, Venturini CL, Ramalho AFS, Santos RAN, Ferrari M. Avaliação in vitro da atividade fotoprotetora de extratos vegetais do cerrado de Mato Grosso. Rev Bras Farmacogn 2009; 19(2): 452-457. DOI: $10.1590 / \mathrm{S} 0102-695 X 2009000300020$

35. Savian AL, Varella FT, Athayde ML, Silva CB. Desenvolvimento e avaliação preliminar da estabilidade de emulsão não-iônica $\mathrm{O} / \mathrm{A}$ contendo óleo de café verde como potencializador de fator de proteção solar. Rev Bras Farm. 2010; 91(2):24-30.

36. Munhoz VM, Lonni AASG, Mello JCP, Lopes GC. Avaliação do fator de proteção solar em fotoprotetores acrescidos com extratos da flora brasileira ricos em substâncias fenólicas. Rev Bras Cienc Farm 2012; 33(2): 225-232.

37. Mansur JS, Breder MNR, Mansur MCA, Azulay RD. Correlação entre a determinação do fator de proteção solar em seres humanos e por espectrofotometria. An Bras Dermatol 1986b;61(4):167-172.

38. Ferrari, M. Desenvolvimento e avaliação da eficácia fotoprotetora de emulsões múltiplas contendo metoxicinamato de etilexila e óleo de andiroba (Caropa guyanensis) [Tese]. São Paulo: Faculdade de Ciências Farmacêuticas de Ribeirão Preto, Universidade de São Paulo; 2002.

39. Adad MCBT. Determinação do Fator de Proteção Solar "In Vitro" [Dissertação]. Porto Alegre: Escola Politécnica, Pontifícia Universidade Católica do Rio Grande do Sul; 2007. 
40. Velasco MVR, Balogh TS, Pedriali CA, Sarruf FD, Pinto CASO, Kaneko TM, Baby AR. Novas metodologias analíticas para avaliação da eficácia fotoprotetora (in vitro) - revisão. Rev Bras Cienc Farm 2010; 32(1): 27-34.

41. Garoli D, Pelizzo MG, Bernardini B, Nicolosi P, Alaibac M. Sunscreen tests: Correspondence between in vitro data and values reported by the manufacturers. J Dermatol Sci 2008; 52(3): 193-204. DOI: 10.1016/j.jdermsci.2008.06.010

42. Schalka S, Addor FAZ, Agelune CM, Pereira VMC. Sunscreen protection against visible light: a new proposal for evaluation. Surg Cosmet Dermatol 2012; 3(4): 4552.

43. Bergold AM, Ponzio HA, Camargo LN, Ribeiro MH, Dias MC, Sartori VLG. Variação do Fator de Proteção Solar (FPS) de acordo com a técnica de preparação de fotoprotetores tópicos. Caderno de Farmácia 1993; 9(2): 77-80.

44. Ribeiro RP. Desenvolvimento e validação da metodologia de análise do teor de filtros solares e determinação do FPS in vitro em formulações fotoprotetoras comerciais [Dissertação]. Rio de janeiro: Faculdade de Farmácia, Universidade Federal do Rio de Janeiro; 2004.

45. Caswell M. Susnscreen formultion and testing. Cosmet Toiletries 2001; 116(9): 49-60.

46. Masson P, Scotti L. Fotoproteção: um desafio para a cosmetologia. Cosmet Toiletries 2003; 15(4): 42-53.
47. Mundstock MH, Frasson APZ. Avaliação da Estabilidade Físico-Química de Bloqueadores Solares FPS 30. Contexto \& Saúde 2005; 5(8): 56-66.

48. Chorilli M, Udo MS, Cavallini ME, Leonardi GR. Desenvolvimento e estudos preliminares de estabilidade de formulações fotoprotetoras contendo Granlux GAI-45 TS. Rev Ciênc Farm 2006; 27(3): 237-246.

49. Melo MR, Siqueira APNDF. Desenvolvimento de uma loção fotoprotetora, avaliação da estabilidade e determinação in vitro do FPS. Perquirere 2012; 9(1): 81-97.

50. Hagedorn LU, Lippold B. Accumulation of suncreens and other compounds in keratinous substrates. Eur J Pharm Biopharm 1998; 46: 215-221. DOI: 10.1016/ S0939-6411(97)00165-3

51. Ojoe E, Luna FP, Cassino S, Cassino F, Mirabelli F. Inovação em Fotoproteção. Rev Pharm Compounding 2004; 6(6): $1-10$.

52. BRASIL. Agência Nacional de Vigilância Sanitária. Resolução - RDC No 30 de $1^{\circ}$ de junho de 2012. Aprova o Regulamento Técnico Mercosul sobre Protetores Solares em Cosméticos e dá outras providências. Maio de 2012. $7 \mathrm{p}$.

53. Schalka S, Steiner D. Consenso Brasileiro de Fotoproteção. Fotoproteção no Brasil - Recomendações da Sociedade Brasileira de Dermatologia. 1 ed. Rio de Janeiro: Sociedade Brasileira de Dermatologia-SBD, 2013. 